\title{
Modeling the Behavior of Flooding on Target Location Discovery in Mobile Ad Hoc Networks
}

\author{
Jia-Chun Kuo and Wanjiun Liao \\ Department of Electrical Engineering \\ National Taiwan University \\ Taipei, Taiwan \\ Email:wjliao@ntu.edu.tw
}

\begin{abstract}
In this paper, we model the behavior of packet forwarding via a multihop path in mobile ad hoc networks. In our analysis, we consider a densely populated network and assume a flooding protocol for packet forwarding. We find that the behavior of packet forwarding in such an environment can be regarded as a dropping a stone into a lake, and then counting the number of ripples moving out from the source (i.e., the center) to the destination. What makes the problem complicated is the node mobility during the packet forwarding. As a result, we cannot solely count the number of ripples in between as the number of hops in the path on which the packet traverses. We then derive the probability distribution function of hop counts for packet forwarding, accounting for node movements. Based on the analytical model, we then evaluate several different types of flooding mechanisms commonly adopted for target searching in ad hoc networks. Compared with existing work, which assumes a snapshot of the network and all nodes are static in the analysis, our analytical framework provides more insights for the study of efficient flooding in mobile ad hoc networks.
\end{abstract}

Keywords: mobile ad hoc networks, multihop packet forwarding, modeling

\section{INTRODUCTION}

A mobile ad hoc network is a multihop wireless network. In such a network, each node in the network plays both roles of a host and a router. Data packets are then relayed via multiple hops, without the support of fixed infrastructure. Nodes may be roaming around during packet forwarding. Whenever a node on the routing path has moved away, the path must be rerouted.

In this paper, we study the behavior of packet forwarding on a multihop path in mobile ad hoc networks. The behavior of packet forwarding in mobile ad hoc networks may be affected by many different factors, such as node mobility, spatial distribution of nodes, the routing algorithm, and network topology. The impacts of all factors on the performance of packet forwarding have been widely studied in the literature [1-6]. In [1], many mobility models are surveyed. In [2], the impact of packet routing protocols on the end-to-end transmission delay is discussed. The authors also claim that node density affects the delay. In [3], the authors find that node degree and connectivity both have impact on the delay, and thus network topology is also an important factor to consider. In addition, they claim that so long as the transmission range of nodes is properly chosen, the network connectivity is ensured.

In [4], both the length and duration of a movement epoch and the direction of travel according to the random waypoint mobility model are proven to be nonuniform and affected not only by the spatial position of the node but also the simulation region. In [5], the node spatial distribution based on the random waypoint model is analyzed. In [6], a forwarding technique, called $\mathrm{GeRaF}$, is proposed based on the geographic location of nodes. The circular intersection area between the transmission ranges of adjacent nodes and the distance of the destination node are used to derive the analytical bounds for the multihop performance of GeRaF. However, the assumption that the geographic location of all nodes is known a priori is not realistic in general.

In this paper, we model the behavior of packet forwarding on a multihop path in mobile ad hoc networks. In particular, we derive the probability distribution function of the number of hops in a multihop path for packet forwarding, allowing node movements. Based on the analytical model, we further evaluate several different types of flooding mechanisms commonly adopted for target searching in ad hoc networks, including blind flooding (e.g., flooding mentioned in [7]), two-tier flooding (e.g., DSR [8]), and expansion-ring flooding (e.g., AODV [9]), with respect to their cost and target searching latency. Compared with [10], which assumes a snapshot of the network and all nodes are static in the analysis, our analytical framework provides more insights for the study of efficient flooding in mobile ad hoc networks, because we consider node mobility and many network parameters (including distance between nodes, transmission radius and the processing time of nodes) together.

The rest of the paper is organized as follows. In Sec. II, the system model and assumptions are described, and an analytical model is derived. In Sec. III, different types of flooding schemes are evaluated based on the analytical model. Finally, the paper is concluded in Sec. IV.

\section{ANALYTICAL MODEL}

\section{A. System Model and Assumptions}

The system we consider is a homogeneous mobile ad hoc network, in which all nodes are equipped with the same transmission capacity and data are forwarded via a multihop path. Each node is continuously roaming. We do not assume a certain mobility model, but capture the moving behavior by means of the circles centered at the initial location of roaming nodes. In other words, the 
circles for those fast-moving nodes grow faster than those slow-moving ones. We consider a densely populated network. Thus, the isolated node problem will not happen. We also don't consider the interference and handoff problems.

In this paper, we model the distance between source and destination in terms of the number of hops for packets to travel, taking node movements into account. Data packets are forwarded via flooding, considering "flooding" being widely used for target searching in the route discovery phase of most on-demand ad hoc routing protocols. The notation used in the analysis is summarized in Table I.

\begin{tabular}{|c|c|}
\hline$a$ & The radius of the transmission range of each node. \\
\hline$p$ & Packet size (bits) \\
\hline$c$ & Link speed (bps) \\
\hline$t_{x}$ & Packet transmission time, $t_{x}=\frac{p}{c}$ \\
\hline$t_{i}$ & $\begin{array}{l}\text { Processing delay at the } i \text { th node counted from the } \\
\text { source on a multihop path }\end{array}$ \\
\hline$L$ & $\begin{array}{l}\text { The random variable representing the initial distance } \\
\text { between the source and the destination, with } \\
\text { probability density function } f_{L}(l) \text {. }\end{array}$ \\
\hline$r_{c}$ & $\begin{array}{l}\text { The growing rate of the circle centered at the } \\
\text { destination }\end{array}$ \\
\hline$H$ & $\begin{array}{l}\text { The random variable representing the number of } \\
\text { hops to be traversed for packet forwarding }\end{array}$ \\
\hline$F_{H}\left(h_{0}\right)$ & The cumulative distribution function of $H$ \\
\hline$P_{H}(k)$ & The probability density function of $H$ \\
\hline
\end{tabular}

TABLE I. NOTATION IN THE ANALYSIS

\section{B. The Hop Counts for Multihop Packet Forwarding}

In our model, we consider a mobile ad hoc network with high node density. In other words, no matter which direction the next hop is, there is always a node there to forward the packet via flooding. Fig. 1 illustrates an example to forward a packet over a multihop path in our system, where $R$ denotes the radius of the transmission range of each node. Interestingly, such phenomena makes the behavior of packet forwarding along a multihop path for a source-destination pair just like dropping a stone into a lake and generating ripples moving out from the source. What interests us is the number of ripples between the source and the destination, which is equal to the number of hops for the packet to traverse in between.

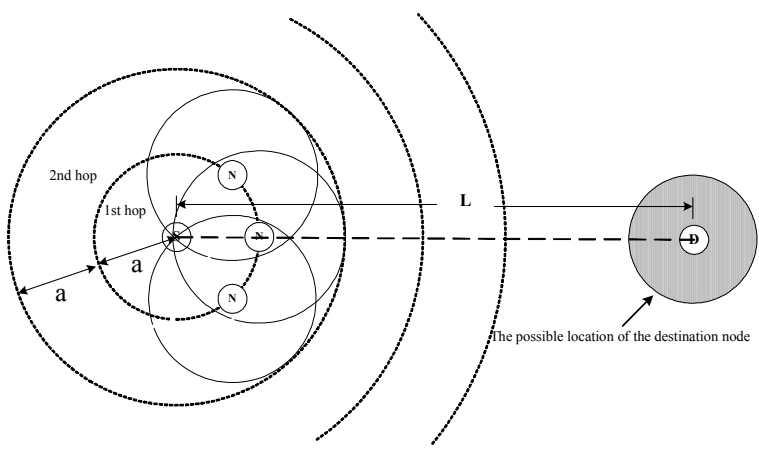

Figure 1. The behavior of multihop packet forwarding

However, this hop count can only be regarded as the initial distance between the source and the destination, because the destination node may be moving around during the packet forwarding. If the destination node is moving towards the source, the final hop count will be smaller than the initial one, and vice verse. What makes this problem complicated is that it is hard to predict which direction the destination node is moving at any moment. The only thing we can be sure is that if the movement speed is higher, the possible area in which the destination node may be located become wider. To capture such an effect, we adopt a circle centered at the destination to represent the possible area in which the destination node may be located at any moment. The higher node mobility then results in a larger circle. As a result, once the moving speed is given, we can predict how the circle grows.

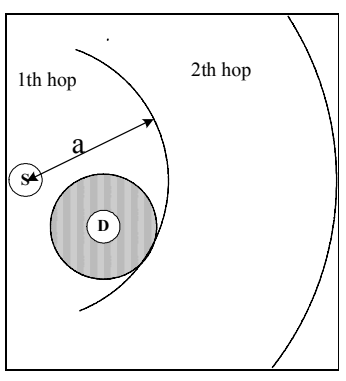

(a) $H \leq 1$

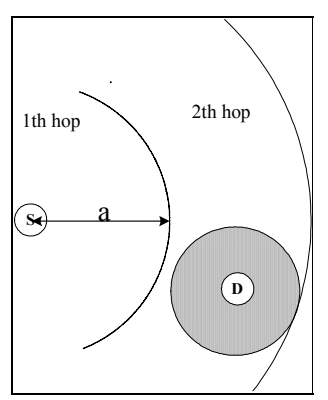

(b) $H \leq 2$
Figure 2. Two examples of packet forwarding

Let $H$ denote the random variable of the hop count for the source-destination pair of interest, and $F_{H}\left(h_{0}\right)$ be the cumulative probability distribution of $H$. Given the initial distance $L$, the number of hops the packet traverses is at most one only if the following condition holds (as shown in Fig. 2 (a)):

$$
a \geq L+r_{c} \cdot t_{x}, \text { i.e., } L \leq \frac{a c-r_{c} p}{c},
$$

where $a$ is the node transmission radius, $r_{c}$ is the circle growing rate, and $t_{x}$ is the packet transmission delay in one hop (the propagation delay is ignored). Thus, the probability with $H$ at most one is 


$$
F_{H}(1)=\operatorname{Pr}(H \leq 1)=\operatorname{Pr}\left(L \leq \frac{a c-r_{c} p}{c}\right)
$$

Now we calculate the probability of the hop count greater than one. Using a two-hop path as an example (Fig. 2 (b)), we have $2 a \geq L+r_{c} \cdot\left(2 t_{x}+t_{1}\right)$, where $t_{1}$ is the processing delay of the first relaying node from the source. Since $t_{x}=\frac{p}{c}$, we obtain $t_{1} \leq \frac{2 a c-L c-2 r_{c} p}{r_{c} c}$. In addition, $0 \leq t_{1}$, thus we have $\frac{2 a c-L c-2 r_{c} p}{r_{c} c} \geq 0$. Due to $r_{c} c \geq 0,2 a c-L c-2 r_{c} p \geq 0$ must hold, i.e., $L \leq \frac{2 a c-2 r_{c} p}{c}$. Thus, the probability that the packet traverses at most two hops is expressed as

$$
\begin{aligned}
& F_{H}(2)= \\
& \frac{2 a c-2 r_{c} p}{c} \\
& \int_{0}^{c} P\left(t_{1} \leq \frac{2 a c-l_{0} c-2 r_{c} p}{r_{c} c} \mid L=l_{0}\right) P\left(L=l_{0}\right) d l_{0}
\end{aligned}
$$

Similarly, the packet traversed at most $N$ hops (i.e., $N-1$ intermediate relaying nodes) only when the following condition holds: $a N \geq L+r_{c} \cdot\left(N \cdot t_{x}+\sum_{i=1}^{N-1} t_{i}\right)$, where $t_{i}$ is the processing delay of the $i$ th relaying node counting from the source on the path, $i=1,2, \cdots, N-1 . \quad$ Substituting $\quad t_{x}=\frac{p}{c} \quad$ into the inequality, we obtain $\sum_{i=1}^{N-1} t_{i} \leq \frac{N a c-L c-N r_{c} p}{r_{c} c}$. Since $0 \leq \sum_{i=1}^{N-1} t_{i}$, we get $L \leq \frac{N a c-N r_{c} p}{c}$. Thus, the probability of at most $N$-hop traveling is expressed as

$$
\begin{aligned}
& F_{H}(N)= \\
& \quad \frac{N a c-N r_{c} p}{c} P\left(\sum_{i=1}^{N-1} t_{i} \leq \frac{N a c-l_{0} c-N r_{c} p}{r_{c} c} \mid L=l_{0}\right) P\left(L=l_{0}\right) d l_{0} .
\end{aligned}
$$

Assume that $\left\{t_{i}: i=1,2, \cdots\right\}$ is a set of independent, identically distributed random variables with probability density function $f(t)$. Let $f_{L}\left(l_{0}\right)$ be the probability density function of $L$. The probability distribution function that the packet can travel at most $N$ hops $F_{H}(N)$ can be further expressed as

$$
\frac{1}{2 \pi j} \int_{\sigma-j \infty}^{\sigma+j \infty \infty} \frac{\left[f^{*}(s)\right]^{N-1}}{s} \cdot\left[e^{\frac{s\left(N a c-N r_{c} p\right)}{r_{c} c}} \cdot \int_{0}^{\frac{N a c-N r_{c} p}{c}} f_{L}\left(l_{0}\right) \cdot e^{-\frac{S l_{0}}{r_{c}}} d l_{0}-\frac{\frac{N a c-N r_{c}, p}{c} \int_{0}^{c} f_{L}\left(l_{0}\right) d l_{0}}{c}\right] d s
$$

where $f^{*}(s)$ is the Laplace transform of $f(t)$.

Let $P_{H}(N), N=1,2, \cdots$, denote the probability of exactly $N$-hop traversal for packet forwarding, i.e., $P_{H}(N)=\operatorname{Pr}(H=N)$. Therefore,

$$
P_{H}(N)=\left\{\begin{array}{lr}
F_{H}(N), & N=1 \\
F_{H}(N)-F_{H}(N-1), & N \geq 2 \\
0, & \text { otherwise }
\end{array} .\right.
$$

\section{THE MODEL VERIFICATION}

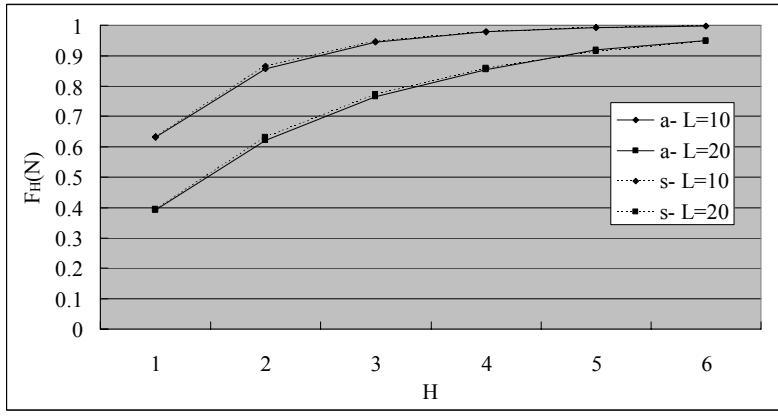

Figure 3. The analytical and simulation results for $F_{H}(N)$

To validate the analytical model by simulations, we plot the cumulative distribution function $F_{H}(N)$ with two different settings of $L$. The settings of other parameters are: $t_{i}=1 \mathrm{sec}, a=10$ units and $r_{c}=0.5$ units per sec. As shown in Fig.3, the analytical and simulation results fit very well.

\section{THE PERFORMANCE OF DIFFERENT FLOODING SCHEMES ON TARGET DISCOVERY}

We have modeled the behavior of a packet being flooded from source to destination in a dense mobile ad hoc network. Based on the analytical model, we can evaluate the cost and latency of different flooding schemes for searching a target destination under various system parameters. In what follows, three different types of flooding schemes for searching a target destination are considered.

(1) Blind flooding: the entire network is flooded (e.g., [7]).

(2) Two-tier flooding: the finite-hop neighbors are searched first. If the target is not found, the entire network is flooded. The searching packet for target location in DSR [8] is an example of two-tier 
flooding.

(3) Expansion-ring flooding: the source incrementally enlarges the searching range from an initial value to a predefined threshold. If the target is still not found, the entire network is flooded. The searching packet for target location in AODV [9] is an example of expansion-ring flooding.

Fig. 4 illustrates the use of the three flooding schemes to search a target in a 4-hop ad hoc network centered at node $S$. The searching range of the network is controlled by the "Time-to-Live" (TTL) parameters (in terms of the number of hops) in the packet header. Fig. 4 (a) illustrates blind flooding, in which a searching packet is flooded to the entire network. Fig. 4 (b) shows two-hop two-tier flooding, in which a searching packet is first broadcast with $\mathrm{TTL}=2$. If the target destination is not found, another searching packet is flooded to the entire network. Fig. 4 (c) depicts expansion-ring flooding, in which a searching packet with initial TTL, say, 2 , is first broadcast. In case the target is not found, the TTL is incremented by one and a searching packet is broadcast with the updated TTL. This is repeated until the predefined threshold (say 3 ) is reached. If the search with the threshold TTL still fails, a final searching packet is flooded to the entire network.

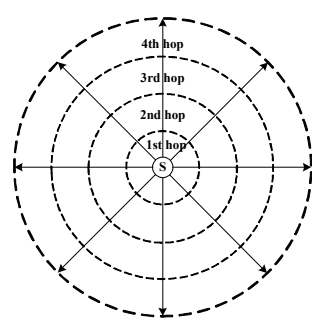

(a)

Blind flooding

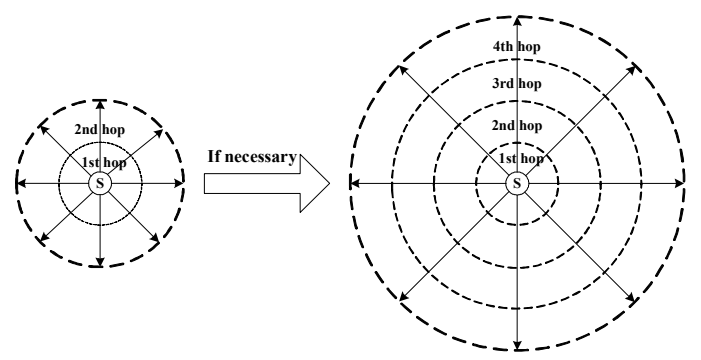

(b) Two-tier flooding

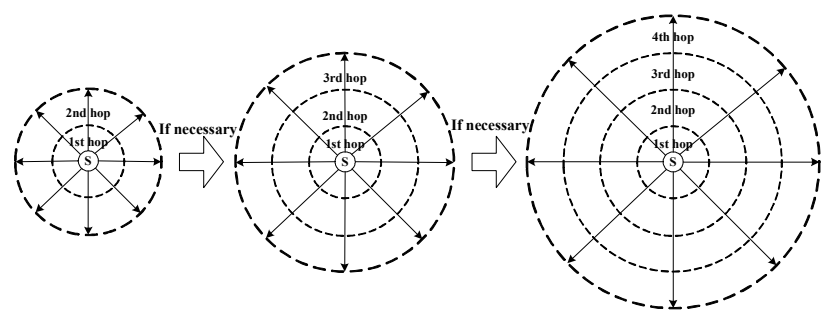

(c) Expansion-ring flooding

Figure 4. Three different flooding schemes

We first evaluate the cost of sending a packet for each flooding scheme, where the cost is defined as the number of times the packet is broadcast by each scheme. A packet is referred to as $k$-hop limited if the packet is propagated up to $k$ hops from the source. The eligible nodes for a $k$-hop limited packet are those located in the area with transmission radius $(k-1) a, k=1,2, \cdots$, but not including those exactly $k$ hops away, from the source. For example, the set of nodes eligible for a 1-hop limited packet include the source node only; the set of nodes eligible for a 2-hop limited packet include the nodes located in the radius of one hop, i.e., $\pi a^{2} d$, where $a$ is the transmission radius and $d$ is the node density. Similarly, the set of nodes eligible for a $k$-hop limited packet includes the nodes located in the area of $(k-1)^{2} \pi a^{2} d, k=1,2, \cdots$. Note that since we consider a dense ad hoc network, $d>>1$.

We consider six different schemes. Scheme 1 represents blind flooding. Schemes 2-4 indicate two-tier flooding with different settings to limit the flooding of the initial packet: for Scheme 2, the first searching packet is one-hop limited, for Scheme 3, the first packet is two-hop limited, and for Scheme 3, the first packet is 3-hop limited. Schemes 5-6 are for expansion-ring flooding with different settings of the initial packet: for Scheme 5, the first packet is one-hop limited, and for Scheme 6 , the first packet is two-hop limited. There is no predefined threshold for Schemes 5 and 6.

Fig. 5 (a) shows the cost of each flooding with different network topologies as shown in Table II, in which each column indicates a different network parameter, and each row shows a different topology setting. The reference row (i.e., Ref) indicates the baseline of comparison for different flooding schemes. Compared to the baseline, row A achieves 33\% reduction in $L$ (i.e., initial distance between source and destination), row B 50\% increase in a (transmission radius of a mobile node), row $\mathrm{C} 80 \%$ reduction in $t_{i}$ (i.e., processing delay of an intermediate node), and row D $80 \%$ reduction in $r_{c}$ (i.e., node mobility). Fig. 5 (b) shows the searching latency of each scheme. From Fig.5, we have some observations.

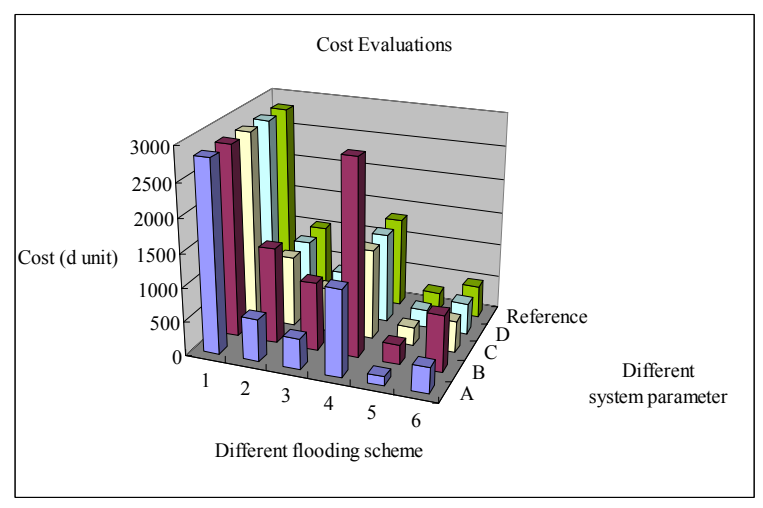

(a) Cost 


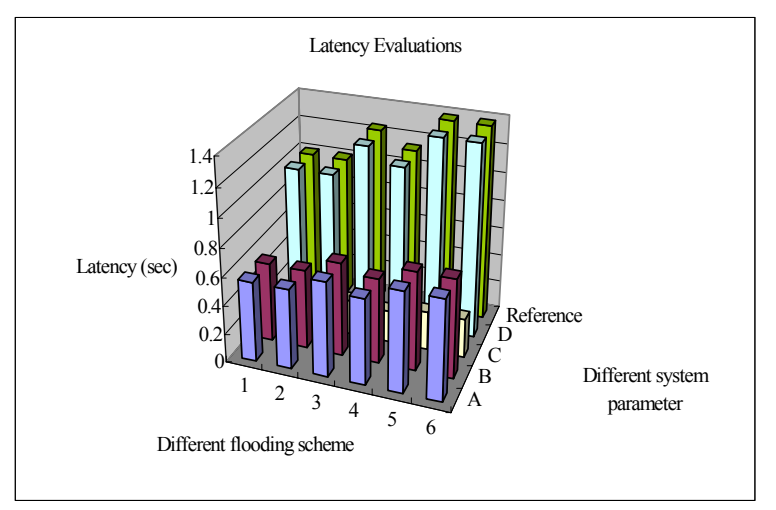

(b) Latency

Figure 5. Performance evaluation

\begin{tabular}{|c|c|c|c|c|}
\hline & L (unit) & $t_{i}$ (sec) & $a$ (unit) & $r_{c}$ (unit/sec) \\
\hline A & 6.67 & 1 & 10 & 0.5 \\
\hline B & 10 & 1 & 15 & 0.5 \\
\hline C & 10 & 0.2 & 10 & 0.5 \\
\hline D & 10 & 1 & 10 & 0.1 \\
\hline Ref & 10 & 1 & 10 & 0.5 \\
\hline
\end{tabular}

TABLE II. NETWORK PARAMETERS

1) Trade-off between the flooding cost and the searching latency

Fig. 5 shows the trade-off between the flooding cost and the searching latency. For blind flooding, the packet is broadcast to the entire network but never retransmitted. Thus, it has the highest flooding cost but the lowest latency. For two-tier flooding, the packet is hop-limited in its first probing, followed by blind flooding if the first trial fails. As a result, it significantly reduces the cost, without dramatically increasing the latency. For expansion-ring flooding, the flooding is done in an incrementally increasing fashion. Thus, it has the lowest cost, but at the expense of high latency due to multiple re-flooding.

2) The impact of different parameters on each flooding scheme

From Fig.5, we learn that only the initial distance between source and destination and the transmission radius of each node are of interest to us. The other parameters affect the metrics of all schemes in a similar way. For example, a reduction in $L$ causes a decrease in the latency for all schemes; similarly, an increase in $a$ decreases the latency of all schemes.

We examine the impacts of a change in $L$ (i.e., row A) and a change in $a$ (i.e., row B) on the flooding cost of all schemes. Blind flooding is immune to the changes in the values of $L$ and $a$, because it always floods to the entire network no matter what happens. For the two-tier and the expansion-ring schemes, the observation is that (a) a reduction in L can lower (and an increase in $a$ can raise) the flooding cost of both types of schemes, and (b) a smaller initial search range saves more flooding cost (see columns 2 and 5 for example, which correspond to the cases of one-hop limited). Such observations match our intuition.

\section{CONCLUSION}

In this paper, we model the behavior of packet forwarding on a multihop path for mobile ad hoc networks with high node density. The node mobility is captured by a circle centered at the initial location of the destination node. Data packets are forwarded via flooding, considering "flooding" being widely used for target searching in the route discovery phase of most on-demand ad hoc routing protocols.

We find that the behavior of multihop packet forwarding can be regarded as dropping a stone into a lake and then counting the number of ripples between the source and the destination as the initial hop count. We then derive the probability distribution functions of hop counts in a multihop path, considering that the destination node may be roaming during packet forwarding. Based on the analytical model, we can then evaluate the cost and latency of different flooding schemes for target discovery.

\section{ACKNOLWDGEMENT}

This paper was supported in part by National Science Council (NSC), Taiwan, under a Center Excellence Grant NSC93-2752-E-002-006-PAE, and in part by NSC under Grant Number NSC93-2213-E-002-132.

\section{REFERENCES}

[1] T.Camp, J. Boleng, and V. Davies, "A Survey of Mobility Models for Ad Hoc Network Research," Wireless Comm. and Mobile Computing (WCMC), vol. 2, no. 5, pp.483-502, 2002.

[2] C.E. Perkins, E.M. Royer, S.R. Das, and M.K. Marina, "Performance Comparison of Two On-Demand Routing Protocols for Ad Hoc Networks," IEEE Personal Comm., Vol.8, Issue 1, pp.16-28, Feb. 2001.

[3] C. Bettstetter, "On the Minimum Node Degree and Connectivity of a Wireless Multihop Network," Proc. ACM MobiHoc, June, 2002.

[4] C. Bettstetter, H. Hartenstein, and Xavier Perez-Costa, "Stochastic Properties of the Random Waypoint Mobility Model: Epoch Length, Direction Distribution, and Cell Change Rate," Proc. ACM MSWiM, Sep. 2002

[5] C. Bettstetter, G. Resta, and P. Santi, "The Node Distribution of the Random Waypoint Mobility Model for Wireless Ad Hoc Networks," IEEE Trans. on Mobile Computing, vol. 2, no. 3, Jul-Sep, 2003.

[6] M. Zorzi and R. R. Rao, "Geographic Random Forwarding (GeRaF) for Ad Hoc and Sensor Networks: Multihop Performance," IEEE Trans. on Mobile Computing, vol. 2, no. 4, Oct-Dec, 2003.

[7] C. Ho, K. Obraczka, G. Tsudik, and K. Viswanath, "Flooding for reliable multicast in multi-hop ad hoc networks," Proc. the International Workshop on Discrete Algorithms and Methods for Mobile Computing and Communication (DIALM), pp. 64-71, 1999.

[8] D. Johnson and D. Maltz, "Dynamic Source Routing in Ad Hoc Wireless Networks," T. Imielinski and H. Korth, Eds. Mobile Computing, Ch. 5, Kluwer, 1996.

[9] C. E. Perkins, E. M. Royer, and S. R. Das, "Ad Hoc on Demand Distance Vector (AODV) Routing," IETF Internet Draft.

[10] Z. Cheng, W. B. Heinzelman, "Ad Hoc and Sensor Networks: Flooding Strategy for Target Discovery in Wireless Networks," Proc. ACM Modeling Analysis and Simulation of Wireless and Mobile Systems, Sep. 2003. 